\title{
PROPIEDADES GEOMÉTRICAS Y MECÁNICAS DEL BLOQUE HUECO DE CONCRETO FABRICADO EN EL ÁREA DE TUXTLA GUTIÉRREZ (CHIAPAS, MEX.)
}

\begin{abstract}
GEOMETRIC AND MECHANICAL PROPERTIES OF HOLLOW CONCRETE BLOCKS MANUFACTURED IN THE AREA OF TUXTLA GUTIÉRREZ (CHIAPAS, MEX)
\end{abstract}

\author{
J. Alejandro Ruiz Sibaja ${ }^{1}$ \\ asibaja@unach.mx \\ Francisco Vidal Sánchez ${ }^{2}$ \\ fvidal@iag.ugr.es \\ Arcadio Zebadúa Sánchez ${ }^{1}$ \\ zebaduaunach@gmail.com
}

1 Facultad de Ingeniería, Universidad Autónoma de Chiapas, México 2 Instituto Andaluz de Geofísica, Universidad de Granada, España 
Para citar este artículo:

Ruiz-Sibaja, J., Vidal, F., Zebadúa, A. (2019). Propiedades geométricas y mecánicas del bloque hueco de concreto fabricado en el área de Tuxtla Gutiérrez (Chiapas, Mex.). Espacio I+D, Innovación más Desarrollo. VIII(21), 8-31. doi: http://dx.doi.org/10.31644/ IMASD.21.2019.a01

\section{RESUMEN}

Se presentan los resultados de una serie de pruebas para la caracterización geométrica y mecánica de bloques de concreto -de 8 fabricantes- y del mortero utilizados en mampostería en la región de Tuxtla Gutiérrez. Los ensayos en laboratorio se realizaron de acuerdo con normas y protocolos de organismos mexicanos. Los resultados de las muestras analizadas revelan que la altura de los bloques es muy variable y difiere de la estándar, mientras que las demás dimensiones y espesores de pared cumplen la normativa. La resistencia a compresión de las probetas del mortero de pega es buena ( 4 veces superior a lo exigido). La absorción total de agua de los bloques es mayor que el máximo permitido ( $12 \%$ ) en la mitad de casos ensayados, pero el peso volumétrico no alcanza, en ningún caso, el mínimo exigido (1700 $\left.\mathrm{kg} / \mathrm{m}^{3}\right)$. La resistencia a compresión de piezas individuales y de pilas de bloques hechas con mortero tipo I es muy inferior a la de la normativa (60 $\mathrm{kg} / \mathrm{cm}^{2}$ y $50 \mathrm{~kg} / \mathrm{cm}^{2}$ respectivamente). La calidad y resistencia deficiente que han mostrado las piezas ensayadas advierten la necesidad de regulación y control local del proceso de fabricación de los bloques.

\section{Palabras Clave}

bloque de concreto; caracterización mecánica; resistencia a compresión; mampostería; normativa.. 
This paper presents the results of a series of tests for geometric and mechanical characterization of concrete block (from 8 local factories) and cement mortar used in masonry in the region of Tuxtla Gutierrez. The laboratory tests were performed according to standards and protocols of Mexican organisms. The results of the analyzed samples show that the height of the blocks is very variable and differs from the standard values while the other dimensions and wall thicknesses comply with the regulations. The compressive strength of the mortar specimens is good (4 times higher than required). The total water absorption of the blocks is greater than the maximum allowed (12\%) in half of cases tested, but the volumetric weight does not reach, in any case, the minimum one required $\left(1700 \mathrm{~kg} / \mathrm{m}^{3}\right)$. The compression strength of individual pieces and block piles (manmade with type I mortar) is much lower than that required in the regulations (6o kg/ $\mathrm{cm}^{2}$ and $50 \mathrm{~kg} / \mathrm{cm}^{2}$ respectively). The quality and poor strength shown by the tests carried out warn of the need for regulation and local control of the block manufacturing process.

\section{Keywords}

Concrete block; mechanical testing; compression resistance; masonry; regulation. 
$\mathrm{E}$ 1 bloque de concreto (вс) es un material básico de construcción de viviendas en México, siendo el más empleado en la construcción de muros de mampostería. En Tuxtla Gutiérrez, al igual que en otras muchas regiones de países en vías de desarrollo, se utilizan sin evaluar en laboratorio las características geométricas y mecánicas, las piezas de BC fabricadas en su entorno, que son las mayoritariamente utilizadas. A menudo se olvida que estas características reales son las que deben tenerse en cuenta, tanto en la fase de proyecto como de ejecución para garantizar una edificación con respuesta estructural adecuada ante distintos tipos de solicitaciones, incluidas las sísmicas en regiones activas.

Una gran parte de las viviendas de interés social que se construyen en México, son edificaciones de uno y dos niveles, a base de muros de mampostería y concreto, que deben soportar cargas verticales y horizontales. En las grandes ciudades, como la Ciudad de México, es común la construcción de edificios de departamentos de 4 o más niveles, para este tipo de viviendas.

En México, la mayoría de las viviendas se construyen a base de muros de mampostería y losas de concreto reforzado. Según el Instituto Nacional de Estadística y Geografía (INEGI) la mayoría de las viviendas son estructuras murarias (de mampostería de ladrillo, вс, tabicón y piedra) que en 1990 eran el 69.5\%, aumentando al 75.6, 78.9 y $79.5 \%$ en 1995, 2000 y 2004 , respectivamente (Castro Hernández et al 2009), y en 2010 representaban el $92.0 \%$ de las viviendas urbanas y el 65.4\% de la vivienda rural (DOF, 2014). En Tuxtla Gutiérrez, el 47\% de las viviendas de interés social se construyen de muros de mampostería de вс (Argüello Méndez et al, 2012).

El вC es un elemento modular, fabricado por moldeo del concreto, que se utiliza tanto en la albañilería estructural, como no estructural. Es un producto compacto, de forma rectangular, tridimensional, de color natural grisáceo y de superficie rugosa, que empezó a usarse en Latinoamérica en la primera década del siglo XX. Poco a poco se ha ido imponiendo en la industria de la construcción, sobre todo por la facilidad de su fabricación y la rapidez en el avance de obra.

El uso de los bloques de concreto supone ciertas ventajas, respecto de materiales tradicionales como el ladrillo o el adobe. Es un material versátil y su uniformidad permite, que las paredes que se levanten sean completamente verticales. Las celdas verticales de los bloques de concreto en los muros son muy útiles porque dentro de ellas se pueden colocar el acero de refuerzo vertical, las instalaciones eléctricas y las hidrosanitarias, entre otras. Esto evita hacer perforaciones en las paredes y acelera la colocación de los sistemas citados, lo que supone un gran ahorro de tiempo y mano de obra.

Las virtudes de este material no terminan aquí, porque si se fabrican o cortan en forma de "U" pueden servir para construir los refuerzos superiores de marcos de puertas y los vanos de las ventanas, también conocidos como 
dinteles. Al utilizar este procedimiento se ahorra tiempo, y una buena cantidad de material. Conviene señalar que los bloques de concreto tienen una magnífica adherencia a los recubrimientos debido a su textura; son de baja absorción, lo que evita una mala adherencia por contracción, y tienen una alta compatibilidad con elementos a base de cemento. Además, se puede p.ej. aumentar su capacidad de aislamiento térmico y acústico rellenando las perforaciones verticales con materiales específicos para ese fin.

Debido al uso extendido de bloques de concreto en edificación, tanto en construcciones a base de muros como en otros tipos de estructuras, la industria de la construcción tiene que producir grandes cantidades de este material para satisfacer esta gran demanda. Se ha encontrado que la resistencia a compresión del ladrillo o del bloque es una de las características estructurales más importantes, en particular la resistencia de piezas de concreto depende principalmente del control de calidad del proceso de fabricación y del nivel de industrialización de la planta (Alcocer, 1997). Por ello, deben establecerse dichos controles de calidad para garantizar la seguridad de los usuarios finales de las construcciones murarias con este material.

A modo de ejemplo citaremos algunas investigaciones realizadas para identificar las características geométricas y mecánicas de la mampostería de вс. La gran mayoría de los estudios experimentales desarrollados en mampostería en el mundo se han dedicado al estudio de las propiedades mecánicas de los materiales. En México, destaca la investigación realizada en los años 70 del siglo pasado por el profesor Meli (1975), que incluyó el estudio de la variabilidad de los materiales componentes, la determinación de propiedades básicas de la mampostería en ensayos de especímenes pequeños, el estudio del comportamiento ante cargas laterales en una dirección y ante cargas alternadas. Este trabajo permitió mejorar las recomendaciones de diseño para estructuras de mampostería en México.

Tena et al (2007) investigaron la respuesta a carga cíclica de muros de mampostería mixta (a base de piezas de ladrillo y de bloques de concreto) y confinada. Realizaron ensayos de la resistencia compresión de piezas de bloque de $12 \times 18 \times 38 \mathrm{~cm}^{3}$ y del mortero de pega conforme al protocolo por las Normas Técnicas para Diseño y Construcción de Estructuras de Mampostería (NTCM-17) y las normas del Organismo Nacional de Normalización y Certificación de la Construcción y Edificación (ONNCCE). Sus resultados indican que las propiedades de los bloques son malas (pobres) en general, y no satisfacen los requisitos de las normas mexicanas, salvo el módulo de ruptura, el cual resultó relativamente alto $\left(f_{\mathrm{r}}=8.14 \mathrm{~kg} /\right.$ $\mathrm{cm}^{2} \cong 0.17 \bar{f}_{\mathrm{p}}$, donde $\bar{f}_{\mathrm{p}}$ es la media de la resistencia a compresión de las piezas, referida al área bruta), mayor al valor mínimo de $5 \mathrm{~kg} / \mathrm{cm}^{2}$ exigido en la NMX-C-404-2012-ONNCCE. El ensayo a compresión del mortero dio una 
resistencia índice de diseño $f_{\mathrm{j}}^{*}=136.6 \mathrm{~kg} / \mathrm{cm}^{2}$, superior a los $125 \mathrm{~kg} / \mathrm{cm}^{2}$ que se establece en la NTCM-17 para un mortero tipo I.

Morales Padilla (2008) investigó la resistencia a compresión de bloques de concreto de la región de Perote, Ver., de acuerdo con las normas del ONNCCE. Se evaluaron especímenes, de $12 \times 20 \times 40 \mathrm{~cm}^{3}$, de tres proveedores de esta región. Los resultados indicaron que ninguno de los proveedores cumple con la resistencia mínima de $60 \mathrm{~kg} / \mathrm{cm}^{2}$ requerida por la normativa, siendo el máximo calculado de resistencia a la compresión promedio de 36 $\mathrm{kg} / \mathrm{cm}^{2}$-sólo un $60 \%$ de lo que exige la normativa-.

Sobre trabajos realizados en otros países latinoamericanos mencionaremos algunos, p.ej. en Perú Quiun et al (2007) ensayaron a compresión axial 60 pilas de albañilería, construidas con piezas de ladrillo y de вс, para determinar experimentalmente los coeficientes de corrección aplicables sobre la resistencia característica $f_{\mathrm{m}}{ }^{\prime}\left(f_{\mathrm{m}}{ }^{*}\right.$ en la normativa mexicana) en pilas que presentan esbelteces distintas al valor nominal que indica la normativa peruana -5 para el Servicio Nacional de Capacitación para la Industria de la Construcción (SENCICO, 2004) y 2 para el Instituto Nacional de Defensa de la Competencia y de la Propiedad Intelectual (INDECOPI, 2003)-. Los resultados indicaron que los coeficientes de corrección eran un poco inferiores a los valores de SENCICO, y más bajos que los de INDECOPI, 2003 -que son iguales a los de la Sociedad Americana de Pruebas de Materiales (AStm, por sus siglas en inglés), (АSTM, 2003)-.

También en Perú, San Bartolomé et al (2007) investigaron la forma de mejorar la adherencia bloque-mortero mediante el empleo de aditivos (en líquido y en polvo). Se realizaron ensayos de compresión axial y de compresión diagonal en pilas de albañilería, y se alcanzaron mejoras significativas en la adherencia cuando se utilizó aditivo en polvo en el mortero, sin haber alterado la resistencia a compresión de la albañilería.

En El Salvador, Arias Guevara et al (2013) realizaron un amplio estudio sobre la calidad del вс mediante ensayos de peso volumétrico, absorción y resistencia a la compresión utilizando piezas de seis proveedores locales. Las pruebas se realizaron según el protocolo Asтм С 90-99a, que indica los requisitos en las propiedades físicas que deben cumplir las piezas de concreto. Para la determinación del control de calidad de los bloques se tomaron muestras de varias fábricas semi-industrializadas, para el muestreo correspondiente. Los resultados mostraron que la resistencia promedio a compresión, de piezas individuales, se encuentra entre 77.93 y $56.74 \mathrm{~kg} /$ $\mathrm{cm}^{2}$, ninguna de las resistencias obtenidas de los bloques elaborados por las fábricas en estudio alcanzó la resistencia mínima exigida por la norma que es de $133 \mathrm{~kg} / \mathrm{cm}^{2}$. Por otra parte, la ASTM C-90 establece una absorción máxima de $240 \mathrm{~kg} / \mathrm{m}^{3}$, que cumplieron la mayoría de las piezas ensayadas, excepto un proveedor que presentó una absorción promedio de $291.16 \mathrm{~kg} /$ 
$\mathrm{m}^{3}$. Por lo que se refiere a la densidad, la Алтм C-90 indica que las piezas con densidad entre 1682 y $2000 \mathrm{~kg} / \mathrm{m}^{3}$ se consideren de peso mediano, así, cinco de los lotes presentaron piezas de peso mediano (con densidades entre 1694.13 y $1948.00 \mathrm{~kg} / \mathrm{m}^{3}$ ) y uno de ellos resultó de peso normal (con densidad de $2079.46 \mathrm{~kg} / \mathrm{m}^{3}$ ).

Navas Carro y Fonseca Mojica (2016) analizaron los resultados obtenidos en estudios previos realizados sobre mampostería de вс en Costa Rica. Para piezas de $12 \times 20 \times 40 \mathrm{~cm}$, encontraron que los valores de la densidad individual de los bloques ensayados son $2067 \mathrm{~kg} / \mathrm{m}^{3}$ (condición seca) y $2243 \mathrm{~kg} / \mathrm{m}^{3}$ (condición saturada), valores considerados como de peso normal según la Astm C-9o.

En Chiapas, a pesar del amplio y atractivo uso de este material, se carece de estudios suficientes, sobre la caracterización geométrica y mecánica del BC fabricado localmente, que permitan realizar un cálculo realista de este tipo de construcciones y alcanzar así niveles convenientes de seguridad estructural. Por otro lado tampoco se dispone de una normativa regional de construcción para diseño de estructuras con este sistema constructivo. El reglamento vigente de construcción para Tuxtla Gutiérrez, no incluye un apartado en el que se aborde el diseño de edificaciones de mampostería o se recomiende la calidad de los elementos de mampuesto usados.

El desconocimiento de las características geométricas y propiedades mecánicas de este material puede conducir a hipótesis de cálculo incorrectas de edificaciones murarias, lo cual puede originar p.ej. la presencia de agrietamientos ante fenómenos extraordinarios, e incluso poner en riesgo su funcionalidad estructural. El estudio de piezas de mampostería no incluye únicamente su capacidad a compresión, también deben evaluarse otras características tales como: geometría, peso volumétrico y absorción de humedad. Además, debe conocerse la capacidad del mortero que se utiliza para pegar los bloques de concreto.

Debido a la ausencia de una normativa específica en Chiapas, en Tuxtla Gutiérrez no existe regulación de la calidad del вс producido por los diferentes fabricantes de la región. Como consecuencia, no hay uniformidad en la fabricación de este material, y por lo general los bloques son de calidad dudosa y deficiente, lo cual, en el caso de una solicitación sísmica extraordinaria, puede poner en riesgo a las estructuras construidas con este sistema y a sus ocupantes.

En este trabajo se presentan los resultados de pruebas controladas en laboratorio en bloques de concreto, provenientes de 8 proveedores diferentes de la ciudad Tuxtla Gutiérrez, y del mortero de pega, para poder estimar el comportamiento de estructuras murarias. Las pruebas se realizaron, de septiembre de 2016 a mayo de 2017, en el Laboratorio de Mecánica de Suelos y Resistencia de Materiales, de la Facultad de Ingeniería de la Universidad Autónoma de Chiapas, de acuerdo con el protocolo indicado en las normas 
NTCM-17 y ONNCCE. Se consideraron los siguientes ensayos: medición de la geometría, absorción total de agua, capacidad del mortero de pega cemento-arena, resistencia a compresión en piezas individuales y en pilas de bloques. Esta es una primera aproximación a las características geométricas y propiedades mecánicas de este material, por lo tanto, únicamente se consideraron pruebas de compresión simple durante los ensayos realizados. En una etapa posterior se realizarán pruebas a cortante o tensión diagonal, adherencia y a flexión, para conseguir una mejor aproximación a la realidad física del bloque hueco de concreto. A continuación se describen los ensayos efectuados y se discuten sus resultados.

\section{ENSAYOS REALIZADOS}

Se preparó el material necesario de acuerdo con las recomendaciones de las normativas mencionadas (NTCM-04, ONNCCE). Así, por cada uno de los 8 lotes de ensayo (con вс provenientes de cada uno de los proveedores) se utilizaron para cada prueba el número de piezas siguiente:

- Caracterización de la geometría: 10 piezas

- Absorción inicial de agua: 10 piezas

- Resistencia a compresión simple: 10 piezas

- $\quad$ Resistencia a compresión de pilas: 3 piezas por pila (se ensayaron 3 pilas).

Por tanto se necesitaban un mínimo de 29 piezas por cada proveedor, pero previendo algún tipo de alteración del material por transporte, manejo u otros imprevistos, se adquirieron varias piezas más por cada lote.

\section{Mortero de pega}

Para realizar las pruebas necesarias del mortero de pega se utilizó cemento para albañilería, con las características que recomiendan las NMX-C021-ONNCCE-2015 Y NMX-C-414-ONNCCE-2017. Los ensayos con piezas de BC, mortero y pilas de вс se realizaron en el Laboratorio de Mecánica de Suelos y Resistencia de Materiales, de la Facultad de Ingeniería de la Universidad Autónoma de Chiapas.

Los ensayos de mortero se llevaron a cabo conforme a la NMX-C464-ONNCCE-2010. Se utilizaron moldes de acero con dimensiones de $5 \times 5 \times 5$ $\mathrm{cm}^{3}$ para preparar las probetas de mortero. En los ensayos realizados se empleó la proporción más utilizada para mortero de pega en la práctica profesional local de 1:3 (cemento-arena, mortero clasificado como tipo I de acuerdo con las NTCM-17). 
La pasta del mortero se preparó considerando las NMX-C-021-ONNCCE-2015 Y NMX-C-061-ONNCCE-2015. Con esta pasta se rellenaron los moldes hasta la mitad, se apisonó el contenido, se rellenaron nuevamente y se enrasó cada molde. En total se realizaron 24 probetas, que se almacenaron durante 3, 7, 14 y 28 días, respectivamente. Al cabo de cada período, se ensayaron a compresión 6 probetas (ver Figura 1), hasta ensayar definitivamente las 24 muestras preparadas. Los resultados para las probetas de 28 días se muestran en la Tabla 1.

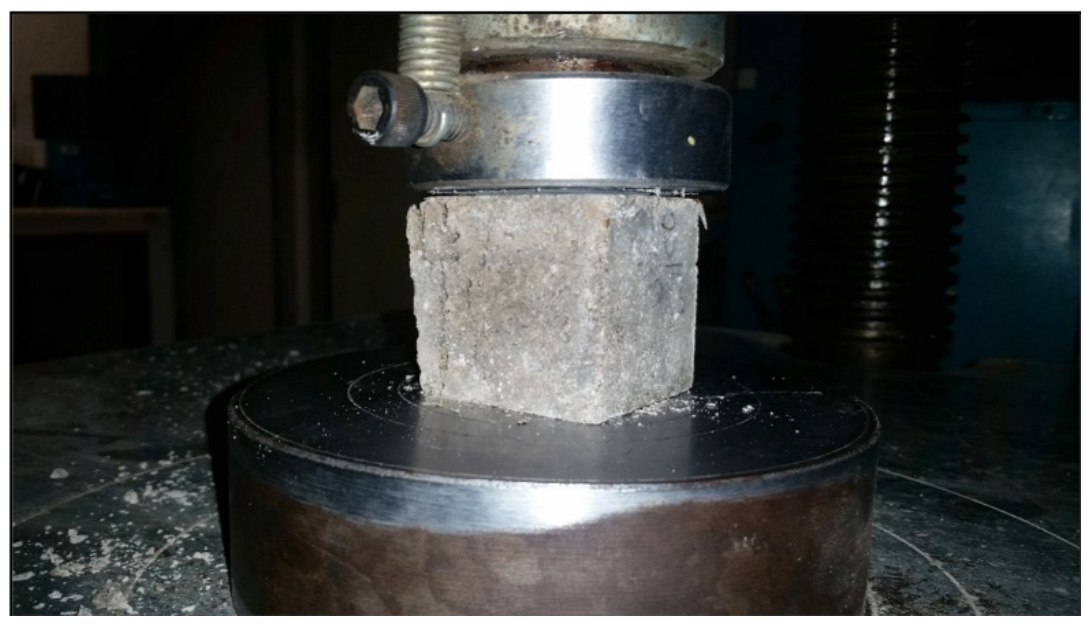

Figura 1. Ensayo de cubo de mortero en prensa universal

\section{Tabla 1}

Resultados de pruebas de compresión de probetas de mortero de 28 días

\begin{tabular}{ccc}
\hline Número de probeta & Carga final $(\mathrm{kg})$ & $\begin{array}{c}\text { Resistencia a compresión } \\
\left(\mathrm{kg} / \mathrm{cm}^{2}\right)\end{array}$ \\
1 & 6321.00 & 245.43 \\
2 & 6295.00 & 246.86 \\
3 & 6348.00 & 253.92 \\
4 & 6290.00 & 246.67 \\
5 & 6268.00 & 250.72 \\
6 & 6353.00 & 249.14 \\
\hline
\end{tabular}

El valor medio de la carga final aplicada (Tabla 1) fue de $6312.50 \mathrm{~kg}$, con una desviación típica $(\sigma)$ de $33.96 \mathrm{~kg}$ y el de la resistencia a compresión fue de $248.79 \mathrm{~kg} / \mathrm{cm}^{2}$, con una $\sigma$ de $3.12 \mathrm{~kg} / \mathrm{cm}^{2}$.

De acuerdo con la NTCM-17 la resistencia de diseño $f_{\mathrm{j}}^{*}$ de las probetas de mortero se calcula con la siguiente expresión: 


$$
f_{j}^{*}=\frac{f_{j}}{1+2.5 C_{j}}
$$

Donde $f_{\mathrm{j}}$ es la resistencia media a compresión de cubos de mortero y $C_{\mathrm{j}}$ es el coeficiente de variación de la resistencia a compresión del mortero, que en ningún caso se tomará menor que 0.20 . La Tabla 2 presenta los resultados de $f_{\mathrm{j}}^{*}$ tras aplicar en la expresión (1) los valores de las resistencias a compresión de la Tabla 1.

\section{Tabla 2}

Resistencia de diseño a compresión de probetas de mortero de 28 días

\begin{tabular}{cc}
\hline Número de probeta & $\begin{array}{c}f_{j}^{*} \\
\left(\mathrm{~kg} / \mathrm{cm}^{2}\right) \\
1\end{array}$ \\
163.62 \\
2 & 164.57 \\
3 & 169.28 \\
4 & 164.45 \\
5 & 167.15 \\
6 & 166.09 \\
\hline
\end{tabular}

Para los datos de la Tabla 2 el valor medio de la resistencia de diseño a compresión y su desviación típica son $165.86 \mathrm{~kg} / \mathrm{cm}^{2}$ y $2.10 \mathrm{~kg} / \mathrm{cm}^{2}$, respectivamente.

\section{Caracterización geométrica de bloques}

Para la caracterización geométrica de los bloques se escogieron 10 bloques por cada proveedor, tal como indica la NMX-C-038-ONNCCE-2013, y se midieron sus dimensiones geométricas con un calibrador "pie de rey" y una regla de apoyo. Se registraron: largo, alto, ancho, espesor de paredes e interior de huecos. No se midieron ni el estriado ni el relieve, ya que las piezas no contaban con estas características. Se obtuvo el promedio y la desviación estándar de las dimensiones registradas para cada lote, así como para todo el conjunto de piezas (ver Tabla 3).

Se midieron también los espesores de pared de las piezas de cada lote, llamando e1 al espesor de las paredes en el sentido longitudinal, y e2 al espesor de las paredes en el sentido transversal. En la Tabla 4 se muestran los valores medios obtenidos de dichos espesores y sus desviaciones típicas. 
Tabla 3

Valor medio y desviación estándar de las dimensiones geométricas de los BC

\begin{tabular}{ccccccc}
\hline Proveedor & Largo $(\mathrm{cm})$ & $\sigma$ largo $(\mathrm{cm})$ & Ancho $(\mathrm{cm})$ & $\sigma$ ancho $(\mathrm{cm})$ & Alto $(\mathrm{cm})$ & $\sigma$ alto $(\mathrm{cm})$ \\
1 & 39.98 & 0.10 & 11.98 & 0.03 & 19.21 & 0.44 \\
2 & 39.90 & 0.15 & 11.93 & 0.08 & 19.90 & 0.13 \\
3 & 39.86 & 0.11 & 11.91 & 0.07 & 18.76 & 0.26 \\
4 & 39.91 & 0.08 & 11.95 & 0.10 & 19.84 & 0.16 \\
5 & 39.82 & 0.16 & 12.02 & 0.08 & 20.09 & 0.35 \\
6 & 40.14 & 0.07 & 12.07 & 0.11 & 19.35 & 0.17 \\
7 & 39.97 & 0.04 & 11.99 & 0.04 & 19.83 & 0.13 \\
8 & 39.97 & 0.06 & 12.00 & 0.04 & 19.89 & 0.15 \\
Todos & 39.94 & 0.14 & 12.00 & 0.04 & 19.61 & 0.49 \\
\hline
\end{tabular}

\section{Tabla 4}

Valor medio y desviación estándar de espesores de pared

\begin{tabular}{ccccc}
\hline Proveedor & e1 $(\mathrm{mm})$ & $\sigma$ e1 $(\mathrm{mm})$ & e2 $(\mathrm{mm})$ & $\sigma \mathrm{e} 2(\mathrm{~mm})$ \\
1 & 26.83 & 0.60 & 25.80 & 0.64 \\
2 & 26.67 & 0.52 & 24.80 & 0.58 \\
3 & 26.91 & 0.52 & 25.00 & 0.52 \\
4 & 27.59 & 1.12 & 25.38 & 0.68 \\
5 & 31.96 & 0.83 & 29.26 & 0.68 \\
6 & 41.25 & 1.88 & 28.98 & 0.78 \\
7 & 27.02 & 0.34 & 27.60 & 0.28 \\
8 & 30.28 & 1.20 & 31.17 & 0.44 \\
Todos & 29.81 & 4.81 & 27.25 & 2.30 \\
\hline
\end{tabular}

\section{Absorción de agua}

Se llevó a cabo la prueba de absorción volumétrica de agua (ver Figura 2) según la NMX-C-404-ONNCCE-2005. Las muestras, previamente identificadas por su procedencia y número de pieza, se secaron cuidadosamente y se pesaron. A este peso se le denomina $\boldsymbol{M s}$ (masa seca del espécimen). Posteriormente los especímenes se sumergieron en agua a la temperatura que indica la norma, entre $17^{\circ}$ y $23^{\circ}$, por un período de 24 horas. Al cabo de 24 horas se sacaron del recipiente en que se depositaron, y se eliminó el agua en todas las superficies del bloque (caras, huecos y paredes). A continuación, se volvieron a pesar, y a este peso se le denomina Msss (masa saturada y superficialmente seca).

Con los datos obtenidos de Ms y de Msss se calculó la absorción volumétrica (A) en porcentaje en 24 horas con la siguiente expresión: 


\section{RESULTADOS}

Para analizar el desempeño de los agentes químicos, se tomaron como control las características de plasticidad original del material (tabla 1). Solo se monitorizaron estas propiedades (límite líquido, límite plástico e índice de plasticidad) debido a que la estabilización del suelo busca disminuir las características de plasticidad del material, ya que es bien sabido que al disminuir éstas, mejoran las características de resistencia y estabilidad volumétrica del suelo.

A continuación, se presentan los resultados de la determinación del límite líquido (figura 3), límite plástico (figura 4) y del índice de plasticidad (figura 5) en todas las muestras adicionadas con los agentes estabilizadores.

$$
\boldsymbol{A}=\frac{M s s s-M s}{M s} \times 100
$$

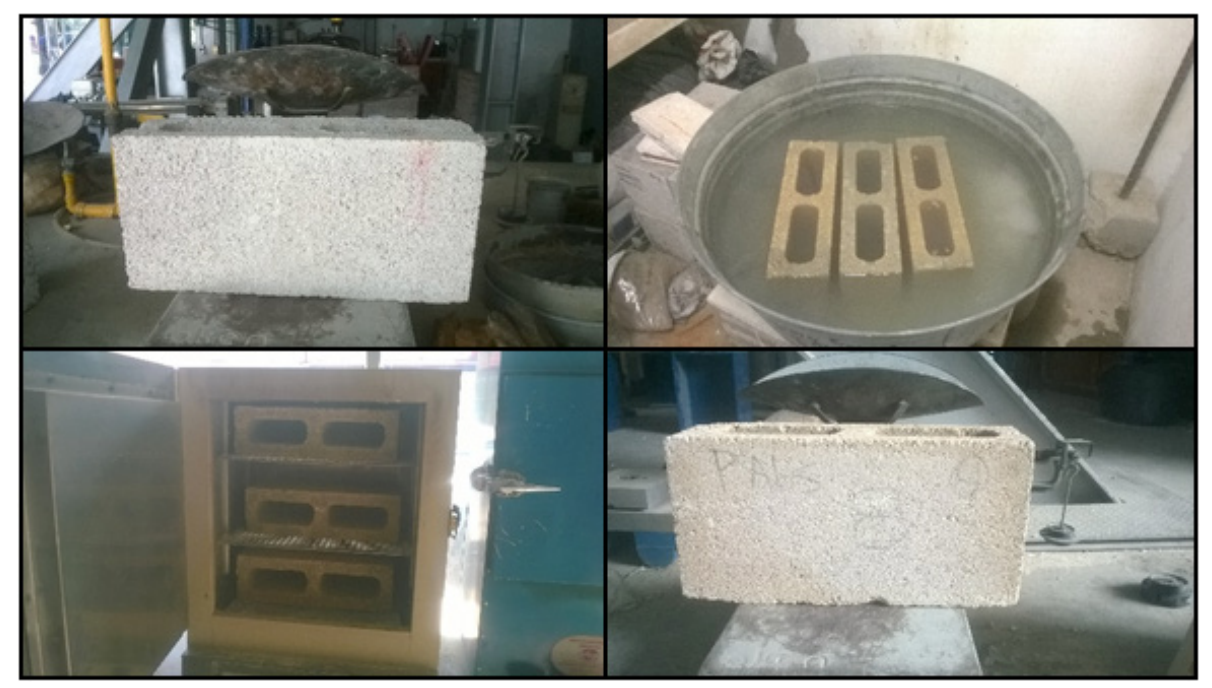

Figura 2. Fotografías que muestran las distintas etapas del ensayo de absorción de agua

En la Tabla 5 se muestran los valores medios de absorción de agua para cada lote y del total de piezas y sus desviaciones estándar. 
Tabla 5

Valor medio y desviación estándar de la absorción de agua

\begin{tabular}{ccccccc}
\hline Proveedor & $\begin{array}{c}\text { Peso seco } \\
\text { promedio } \\
(\mathbf{k g})\end{array}$ & $\begin{array}{c}\boldsymbol{\sigma} \text { seco } \\
(\mathbf{k g})\end{array}$ & $\begin{array}{c}\text { Peso saturado } \\
\text { promedio } \\
(\mathbf{k g})\end{array}$ & $\begin{array}{c}\boldsymbol{\sigma} \text { saturado } \\
(\mathbf{k g})\end{array}$ & $\begin{array}{c}\text { \% de } \\
\text { humedad } \\
\text { promedio }\end{array}$ & $\begin{array}{c}\boldsymbol{\sigma} \text { humedad } \\
\mathbf{( \% )}\end{array}$ \\
1 & 11.90 & 0.21 & 12.94 & 0.19 & 8.81 & 2.31 \\
2 & 11.97 & 0.20 & 12.84 & 0.28 & 7.27 & 1.15 \\
3 & 12.34 & 0.32 & 13.53 & 0.38 & 9.61 & 1.69 \\
4 & 11.68 & 0.32 & 12.67 & 0.23 & 8.55 & 1.64 \\
5 & 12.28 & 0.23 & 14.39 & 0.28 & 17.15 & 1.10 \\
6 & 11.96 & 0.17 & 13.49 & 0.38 & 12.74 & 2.27 \\
7 & 11.71 & 0.21 & 12.84 & 0.24 & 9.65 & 3.29 \\
8 & 12.82 & 0.13 & 14.04 & 0.12 & 9.56 & 2.76 \\
Todos & 12.08 & 0.42 & 13.34 & 0.65 & 10.42 & 3.29 \\
\hline
\end{tabular}

Peso volumétrico

El peso volumétrico de cada bloque se obtuvo dividiendo su peso por el volumen de la pieza. Las NTCM-17 indica los pesos específicos mínimos para cada tipo de pieza de mampostería, que en el caso del BC debe ser de 1700 $\mathrm{kg} / \mathrm{m}^{3}$. Los pesos específicos medios -y sus desviaciones típicas- de cada lote y del total de la población muestreada, se indican en la Tabla 6.

\section{Tabla 6}

Valor medio y desviación estándar del peso volumétrico

\begin{tabular}{ccc}
\hline Proveedor & $\begin{array}{c}\text { Peso Volumérico } \\
\left(\mathrm{kg} / \mathrm{m}^{3}\right)\end{array}$ & $\begin{array}{c}\sigma \text { peso volumétrico } \\
\left(\mathrm{kg} / \mathrm{m}^{3}\right)\end{array}$ \\
1 & 1239.06 & 22.16 \\
2 & 1246.36 & 21.13 \\
3 & 1285.42 & 33.20 \\
4 & 1216.15 & 32.87 \\
5 & 1279.17 & 24.21 \\
6 & 1245.83 & 18.18 \\
7 & 1219.79 & 21.80 \\
8 & 1334.90 & 13.68 \\
Todos & 1258.33 & 43.86 \\
\hline
\end{tabular}

Compresión de piezas individuales

Las pruebas de compresión, tanto de piezas individuales como de pilas (NMX-C-404-ONNCCE-2005, NMX-C-464-ONNCCE-2010, NMX-C-036-ONNC- 
CE-2013), requieren de la preparación de un cabeceo. Éste es un proceso de modificación de la superficie de contacto, que es necesario para que la carga aplicada se distribuya uniformemente en toda el área de contacto entre el bloque y la prensa. El cabeceo debe hacerse en ambas caras de la pieza (ver Figura 3). En la Tabla 7 se dan los valores promedio de carga de ruptura y esfuerzo de compresión junto con sus desviaviones típicas de las pruebas de compresión realizadas en piezas individuales.

\section{Tabla 7}

Valor medio y desviación estándar de carga de ruptura y esfuerzo de compresión en piezas individuales

\begin{tabular}{ccccc}
\hline Proveedor & $\begin{array}{c}\text { Carga de ruptura } \\
(\mathrm{kg})\end{array}$ & $\begin{array}{c}\sigma \text { carga } \\
(\mathrm{kg})\end{array}$ & $\begin{array}{c}\text { Esfuerzo de } \\
\text { compresión } \\
\left(\mathrm{kg} / \mathrm{cm}^{2}\right)\end{array}$ & $\begin{array}{c}\sigma \text { esfuerzo } \\
\left(\mathrm{kg} / \mathrm{cm}^{2}\right)\end{array}$ \\
1 & 6524.60 & 1738.38 & 13.62 & 3.61 \\
2 & 14307.90 & 2839.70 & 30.06 & 5.98 \\
3 & 8574.80 & 2013.00 & 18.06 & 4.20 \\
4 & 9253.20 & 3016.09 & 19.39 & 6.27 \\
5 & 6091.60 & 296.44 & 12.73 & 0.61 \\
6 & 25089.70 & 413.06 & 51.80 & 1.08 \\
7 & 31415.20 & 717.12 & 65.56 & 1.64 \\
8 & 24896.30 & 530.66 & 51.91 & 1.19 \\
Todos & 15769.16 & 9501.53 & 32.89 & 19.73 \\
\hline
\end{tabular}

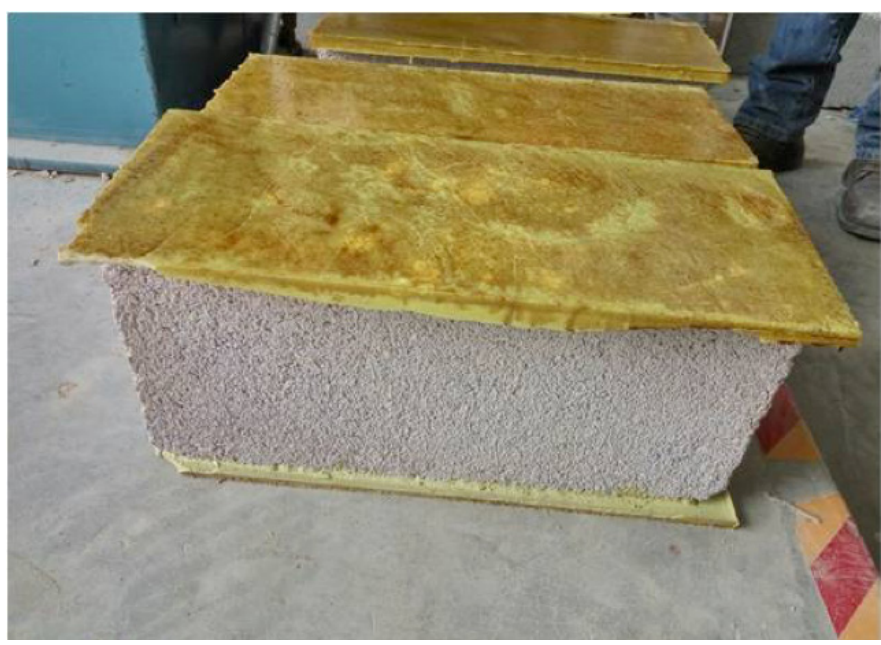

Figura 3.Piezas individuales con cabeceo de azufre

Las NTCM-04 indican que la resistencia de diseño de piezas individuales debe calcularse según la expresión: 


$$
f_{p}^{*}=\frac{f_{p}}{1+2.5 C_{p}}
$$

Donde $f_{\mathrm{p}}$ es la media de la resistencia a compresión de las piezas, referida al área bruta y $C_{p}$ es el coeficiente de variación de la resistencia a compresión de las piezas; el valor de $C_{p}$ no se tomará menor que 0.35 para piezas de producción artesanal. Las resistencias de diseño se calcularon mediante la expresión 3. En la Tabla 8 se dan los valores medios y sus deviaciones típicas.

\section{Tabla 8}

Valor medio y desviación estándar de la resistencia de diseño a compresión de piezas individuales

\begin{tabular}{ccc}
\hline Proveedor & $\boldsymbol{f}_{\mathrm{p}}{ }^{*}\left(\mathrm{~kg} / \mathrm{cm}^{2}\right)$ & $\sigma \boldsymbol{f}_{\mathrm{p}}{ }^{*}\left(\mathrm{~kg} / \mathrm{cm}^{2}\right)$ \\
1 & 7.26 & 1.93 \\
2 & 16.03 & 3.19 \\
3 & 9.63 & 2.24 \\
4 & 10.34 & 3.34 \\
5 & 6.79 & 0.33 \\
6 & 27.63 & 0.58 \\
7 & 34.97 & 0.87 \\
8 & 27.69 & 0.63 \\
Todos & 17.54 & 10.52 \\
\hline
\end{tabular}

Compresión de pilas

Se construyeron pilas con tres piezas tal como indica la NMX-C-464-ONNCCE-2010. A las piezas de los extremos se les aplicó un cabeceo, los bloques se unieron con mortero de pega con proporción 1:0:3, común en la práctica local. En la Figura 4 se muestra como ejemplo una de las pilas sometidas a compresión. 


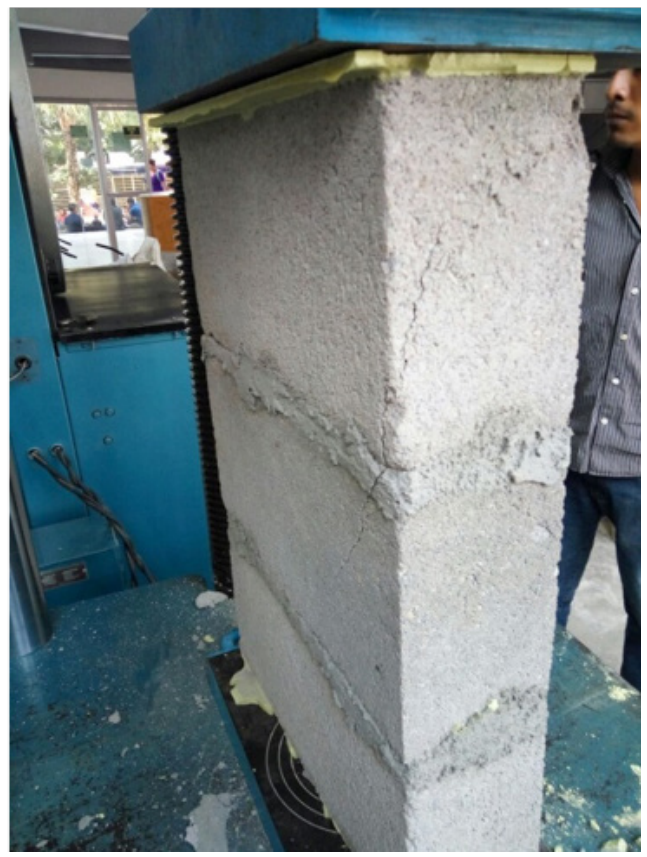

Figura 4.Ensayo de compresión en pila de bloques

Los especímenes se sometieron a carga de compresión hasta la ruptura y se calculó para cada pila el esfuerzo correspondiente. A partir de este esfuerzo se obtuvo la resistencia corregida a compresión de la pila $f_{\mathrm{m}}$ de acuerdo con:

$$
f_{m}=\frac{P}{t \times b} \times \text { factor correctivo por esbeltez }
$$

En la expresión anterior $f_{\mathrm{m}}$ es la resistencia corregida a compresión de la pila (en $\mathrm{MPa}$ o en $\mathrm{kg} / \mathrm{cm}^{2}$ ), $P$ es la carga máxima aplicada (en $\mathrm{N}$ o en $\mathrm{kg}$ ), $t$ es el espesor de la pila (en $\mathrm{mm}$ o $\mathrm{cm}$ ), $b$ es el ancho de la pila (en $\mathrm{mm} \mathrm{o} \mathrm{cm).} \mathrm{El}$ factor depende de la relación de esbeltez de la pila, la cual se calcula como el cociente de entre la altura de la pila y la menor dimensión de la sección transversal, en este trabajo se utilizó un factor correctivo de 1.05, -que corresponde a una relación de esbeltez de 5- de acuerdo con las NTCM-17.

Las NTCM-17 también señalan que el valor de la resistencia de diseño a compresión se calcule a partir de la siguiente expresión:

$$
f_{m}^{*}=\frac{f_{m}}{1+2.5 C_{m}}
$$


En la cual $f_{\mathrm{m}}{ }^{*}$ es la resistencia de diseño a compresión y $C_{\mathrm{m}}$ es un coeficiente de variación de la resistencia a compresión de las pilas de mampostería, que en ningún caso se tomará inferior a 0.15. La Tabla 9 presenta los valores promedio y sus desviaciones típicas de carga de ruptura, resistencia corregida y resistencia de diseño a compresión en pilas.

\section{Tabla 9}

Valor medio y desviación estándar de carga de ruptura, resistencia corregida y resistencia de diseño a compresión en pilas

\begin{tabular}{ccccccc}
\hline Proveedor & $\begin{array}{c}\text { Carga de } \\
\text { ruptura }(\mathrm{kg})\end{array}$ & $\begin{array}{c}\sigma \text { Carga } \\
(\mathrm{kg})\end{array}$ & $\boldsymbol{f}_{m}\left(\mathrm{~kg} / \mathrm{cm}^{2}\right)$ & $\begin{array}{c}\sigma \boldsymbol{f}_{\mathbf{m}}(\mathrm{kg} / \\
\left.\mathrm{cm}^{2}\right)\end{array}$ & $\begin{array}{c}\sigma \boldsymbol{f}_{\mathbf{m}}^{*}(\mathrm{~kg} / \\
\left.\mathrm{cm}^{2}\right)\end{array}$ & $\begin{array}{c}\sigma \boldsymbol{f}_{\mathbf{m}}^{*}(\mathrm{~kg} / \\
\left.\mathrm{cm}^{2}\right)\end{array}$ \\
\hline 1 & 5043.67 & 488.46 & 11.08 & 1.06 & 8.06 & 0.77 \\
3 & 3789.33 & 386.16 & 8.42 & 0.98 & 6.12 & 0.71 \\
4 & 5176.67 & 412.92 & 11.35 & 0.77 & 8.25 & 0.56 \\
5 & 4565.00 & 430.69 & 10.08 & 0.91 & 7.33 & 0.66 \\
6 & 3583.00 & 32.19 & 7.79 & 0.05 & 5.67 & 0.04 \\
7 & 11266.00 & 380.25 & 24.27 & 0.57 & 17.65 & 0.41 \\
8 & 25975.67 & 567.18 & 56.23 & 0.59 & 40.89 & 0.43 \\
Todos & 16898.67 & 189.22 & 36.75 & 1.04 & 26.73 & 0.76 \\
\hline
\end{tabular}

\section{DISCUSIÓN DE RESULTADOS}

\section{Calidad del mortero de pega}

Conviene recordar que la principal función del mortero de pega es proporcionar una buena adherencia de las piezas de mampostería, lo cual se alcanza con una buena dosificación, tal que proporcione adecuada consistencia, suficiente resistencia (a compresión y flexión) y una apropiada capacidad para retener el agua. A su vez, la resistencia a compresión del mortero depende de la relación agua-cemen $\neg$ to y muy especialmente de la granulometría de la arena, la cual se establece mediante el módulo de finura.

Las pruebas realizadas presentan un aceptable comportamiento del mortero ensayado. En las NTCM-17 y la NMX-C-464-ONNCCE-2010, se indica que la resistencia mínima necesaria a compresión, del mortero de pega, debe ser de $40 \mathrm{~kg} / \mathrm{cm}^{2}$. Los resultados encontrados (ver Tabla 2) muestran que el mortero considerado cumple satisfactoriamente con lo que exige la normativa vigente. Se observa que este material es de buena calidad (mortero tipo I de acuerdo con las NTCM-17), y que es adecuada la proporción 1:3 que se utiliza en la práctica local. 
Características geométricas de los bloques

La regularidad de las dimensiones geométricas de los bloques de concreto es una característica que depende en gran medida del proceso de fabricación. Esta uniformidad facilita la determinación de propiedades representativas tales como: área neta, densidad y momento de inercia; además de facilitar cálculos de capacidad a cortante y flexión, así como la determinación de la rigidez. Por ende, estas propiedades son indispensables para el diseño de estructuras de mampostería.

Las dimensiones promedio de las piezas ensayadas de cada uno de los lotes no presentaron desviaciones significativas en longitud y anchura (ver Tabla 3) respecto de las dimensiones de fabricación $12 \times 19 \times 40 \mathrm{~cm}^{3}$ que considera la NMX-C-404-ONNCCE-2005. Sin embargo, hay que destacar que la altura de los вс si presentó desviaciones significativas, además es diferente en cada proveedor (falta de regularidad), y su valor difiere en más de 5 mm la altura estándar de fabricación de dicha norma, estando su valor más cercano al indicado para las dimensiones modulares de los bloques $\left(12 \times 20 \times 40 \mathrm{~cm}^{3}\right.$ que incluye la junta de albañilería de $\left.10 \mathrm{~mm}\right)$.

Esta misma norma señala que para piezas con estas dimensiones el espesor mínimo de las paredes del вс debe ser de $20 \mathrm{~mm}$, propiedad que se cumple en todas las piezas evaluadas (todos los espesores superan dicho valor mínimo) y además la dispersión respecto de la media es pequeña en cada lote (ver Tabla 4). Sin embargo, debe recordarse que la NMX-C038-ONNCCE-2013 indica que en el caso de paredes de bloques expuestas a la intemperie sin recubrimiento, el espesor mínimo debe ser de $30 \mathrm{~mm}$ en un $90 \%$ del área de dicha cara, por lo tanto, si los bloques ensayados se utilizaran para construir paredes sin repello, no se cumpliría con esta normativa.

Conviene señalar que las piezas ensayadas tienen una relación promedio $\mathrm{A}_{\text {neta }} \geq 0.56 \mathrm{~A}_{\text {bruta }}$, que cumple con lo que señala la NMX-C-441-ONNCCE-2013 para piezas de uso no estructural $\left(\mathrm{A}_{\text {neta }} \geq 0.4 \mathrm{~A}_{\text {bruta }}\right)$, y cumple ajustadamente con la NMX-C-404-ONNCCE-2012 la cual, para piezas de uso estructural exige que $\mathrm{A}_{\text {neta }} \geq 0.50 \mathrm{~A}_{\text {bruta }}$.

Estos resultados indican que los bloques ensayados presentan variaciones apreciables en altura (ante la normativa vigente), y por tanto en su geometría, y tienen una falta de regularidad entre fabricantes ya que en el proceso de fabricación se usan moldes de dimensiones no estándar.

\section{Nivel de absorción de agua}

La absorción de agua de los bloques de concreto es una propiedad importante porque está relacionada con la contracción y, en cierta medida con la durabilidad de la pieza. Su importancia también radica en que ésta influye 
directamente en la adherencia entre bloque y mortero, ya que en caso de ser elevada, el agua de amasado del segundo desaparece antes que se produzca una hidratación suficiente del cemento, resultando en una pérdida parcial o total de dicha adherencia y de la resistencia del propio mortero.

La NMX-C-404-ONNCCE-2005 recomienda que para bloques de concreto, la absorción máxima debe ser del $12 \%$. Este grado de permeabilidad, dependiente del proceso de fabricación de las piezas (vibrado, compactado, curado forzado, controlado), beneficia la durabilidad de la pieza y la adherencia entre bloque y mortero.

Los resultados de los ensayos (Tabla 5) indican que las piezas de solamente la mitad de los proveedores cumplen con lo que indica la norma. En el caso de los proveedores 5 y 6 , los bloques absorben cantidades de agua que están muy por encima de lo que señala dicha norma, 17.15 \% y 12.74 $\%$, con $\boldsymbol{\sigma}$ humedad de $1.10 \%$ y $2.27 \%$, respectivamente, y en el caso de los proveedores 7 y 8 , aunque el valor medio de absorción es algo menor del 10 $\%$ su $\boldsymbol{\sigma}$ humedad del $3.29 \%$ y $2.76 \%$, respectivamente, indica que algunas de las piezas tienen una porosidad ligeramente superior a lo recomendado.

\section{Peso volumétrico}

El peso volumétrico es un índice de calidad que indica cuánto espacio ocupa el agregado en la mezcla de concreto; y esta característica puede utilizarse para separar el material bueno del malo. Un bloque de buena calidad se fabrica con una proporción adecuada de cemento y un tiempo suficiente de vibrado y compactado, que hace que se eleve aún más el nivel de resistencia estructural, confiriéndole además una mayor densidad, menor absorción de humedad y mejor calidad de textura superficial. También influye la relación agua-cemento del concreto, debido a que cuanto mayor sea ésta más porosa será la pasta con que se fabrique el bloque.

Los pesos volumétricos medios calculados de los bloques de concreto son menores de $1340 \mathrm{~kg} / \mathrm{m}^{3}$ en todos los lotes (Tabla 6), lo cual advierte que ninguno de los proveedores cumple con el valor mínimo de $1700 \mathrm{~kg} / \mathrm{m}^{3}$ exigido por la NTCM-04. Este déficit indica que las piezas estudiadas son demasiado porosas y que esta deficiencia en la compacidad influye en la resistencia a compresión del material ensayado.

\section{Resistencia a compresión de piezas individuales}

La resistencia a la compresión de los bloques de concreto es una característica importante dado que la función básica de la mampostería es soportar cargas de compresión. Esta propiedad depende principalmente de la densidad y composición del вс у su importancia se puede considerar desde dos puntos 
de vista: primero, a mayor resistencia se tiene mayor durabilidad del material bajo condiciones atmosféricas extremas, y segundo, la resistencia de las piezas incide en la de los muros en mayor medida que la del mortero.

Los valores calculados de resistencia a compresión de diseño para piezas individuales son escasos (Tabla 8). Estos valores revelan que ninguna de las piezas ensayadas alcanza la resistencia mínima de $60 \mathrm{~kg} / \mathrm{cm}^{2}$ que establece la NMX-C-404-ONNCCE-2012. Esta pobre resistencia a compresión, que sólo es un $\mathbf{1 1 . 3 2} \%$ (proveedor 5 ) hasta un $58.28 \%$ (proveedor 7 ) de la resistencia mínima exigida, implica que las piezas ensayadas no garantizan en ningún caso una mampostería de buena calidad, dado que los bloques de concreto deben poseer la suficiente resistencia mecánica para asegurar la correcta transmisión de las cargas, garantizar su durabilidad, y con ello asegurar muros resistentes.

Además, la NMX-C-441-ONNCCE-2013 señala que para piezas de uso no estructural la resistencia media debe ser de $35 \mathrm{~kg} / \mathrm{cm}^{2}$, y su resistencia mínima de $28 \mathrm{~kg} / \mathrm{cm}^{2}$, por lo que estas piezas tampoco son admisibles para uso no estructural, salvo las piezas del proveedor 7 que sí cumplen este requisito, estando las de los proveedores 6 y 8 cercanos a este valor de resistencia.

\section{Resistencia a compresión de pilas}

El ensayo a compresión de pilas de albañilería tiene la intención de reproducir de la mejor manera posible las condiciones de trabajo de la mampostería; como consecuencia, se genera información que puede utilizarse para un diseño estructural adecuado de los muros de un edificio, así como para un buen control de calidad de la albañilería. Las probetas ensayadas presentaron falla frágil, con el desarrollo de una grieta vertical que corta a las piezas y al mortero; esta grieta, que comenzó en las piezas y se extendió al mortero, es debida a la expansión lateral causada por la compresión aplicada.

Los resultados alcanzados muestran que la resistencia de diseño a compresión de las pilas (ver Tabla 9) van desde $5.67 \mathrm{~kg} / \mathrm{cm}^{2}$ (proveedor 1) hasta $40.89 \mathrm{~kg} / \mathrm{cm}^{2}$ (proveedor 7 ), valores que están todos por debajo de los $50 \mathrm{~kg} / \mathrm{cm}^{2}$ que recomiendan las $\mathrm{NTCM}^{-17}$ para pilas de $\mathrm{BC}$ fabricadas con mortero tipo I. Pese a la buena calidad del mortero utilizado para su fabricación, este comportamiento está dominado por la insuficiente resistencia a compresión que muestran las piezas individuales ensayadas.

\section{CONCLUSIONES}

En general, los resultados obtenidos en pruebas controladas de bloques de concreto fabricado en Tuxtla Gutiérrez durante 2016 muestran una mala calidad de las piezas ensayadas. Los ensayos revelan que las piezas no 
cumplen con la normativa vigente, con deficiencias destacables en su peso volumétrico y en su resistencia a compresión -tanto de piezas individuales como de pilas-. Además, se ha observado una gran variabilidad de los parámetros medidos entre los lotes procedentes de distintos fabricantes. En otras investigaciones (Tena et al, 2007; Morales Padilla, 2008; Arias Guevara et al 2013) también se determinó que la calidad de los bloques y de la mampostería ensayada no cumple con la normativa de referencia.

Hay que destacar que la resistencia a compresión del mortero de pega cumplió satisfactoriamente, en todos los casos, con lo que se exige en las NTCM-17 y la NMX-C-464-ONNCCE-2010, ya que las probetas presentaron capacidades muy por encima de $40 \mathrm{~kg} / \mathrm{cm}^{2}$, lo que indica que el mortero que sigue la práctica habitual local es de buena calidad.

Las piezas ensayadas no mostraron uniformidad geométrica aceptable en la altura del bloque, que superó en más de $5 \mathrm{~mm}$ la altura de fabricación estándar de la normativa; cada fabricante suministró piezas de diferente altura y además, las piezas de uno de ellos mostraban entre si una gran variación en su altura. No obstante, debe señalarse que el espesor de las paredes de los bloques si superó en todos los lotes, el mínimo especificado en la normativa.

El valor estimado de absorción volumétrica de agua es muy variable entre lotes ensayados, y sólo en la mitad de ellos es menor del $12 \%$ exigible. En los demás casos, la permeabilidad excesiva de los BC (superior al $17 \%$ en un fabricante) indica una pérdida apreciable en la adherencia bloquemortero y una durabilidad menor de esas piezas.

Los valores de peso volumétrico son bajos y no alcanzan, en ningún caso, ni el 78\% del mínimo exigido en las NTCM-17 $\left(1700 \mathrm{~kg} / \mathrm{m}^{3}\right)$. Esto unido a lo anterior, muestra la conveniencia de mejorar el proceso de fabricación, sobre todo el sistema de compactación y vibrado, para reducir la porosidad actual. Además, para reducir o eliminar estas deficiencias, los fabricantes deberían realizar una gradación adecuada a las arenas que utilizan en la fabricación de bloque (de acuerdo con la NMX-C-077-1997-ONNCCE), con la finalidad que cumplan con la densidad que exige la normativa. Arias Guevara et al (2013) recomiendan utilizar una proporción 1:1.5:1 de cemento-arenagrava para conseguir piezas con absorción óptima, peso volumétrico conveniente y resistencia adecuada a compresión.

Las pruebas de compresión de las piezas individuales mostraron también una resistencia de los bloques muy deficiente y diferente entre fabricantes. Los valores de resistencia de diseño a compresión están entre 6.79 y 34.97 $\mathrm{kg} / \mathrm{cm}^{2}$ muy por debajo de la resistencia mínima de $60 \mathrm{~kg} / \mathrm{cm}^{2}$ que exige la NMX-C-404-ONNCCE-2012.

El ensayo a compresión de pilas también mostró la mala calidad de los bloques para construir estructuras murarias. Los valores calculados de resistencia de diseño a compresión de las pilas son claramente insuficientes, 
entre $5.67 \mathrm{~kg} / \mathrm{cm}^{2}$ y $40.89 \mathrm{~kg} / \mathrm{cm}^{2}$, y están muy por debajo del valor mínimo de $50 \mathrm{~kg} / \mathrm{cm}^{2}$ establecido en las NTCM-04. La buena calidad del mortero utilizado no se tradujo en un mejor comportamiento a compresión de las pilas ensayadas por lo que deben mejorarse las características de resistencia a compresión de los bloques.

Los resultados obtenidos muestran la necesidad de una regulación y control local del proceso de fabricación del bloque de concreto para mejorar la calidad del mismo y asegurar la resistencia de los muros que se fabriquen con este material, condición necesaria en el sector de la construcción local en el cual muchas de las actividades todavía son de carácter artesanal 


\section{REFERENCIAS}

Alcocer, S. M. (1997). Comportamiento sísmico de estructuras de mampostería: una revisión. XI Congreso Nacional de Ingeniería Sísmica, Veracruz, México, pp. 164-191.

Argüello Méndez, T.; Argüelles León, B. y Badillo González, R. M. (2012). Características físicas de la vivienda popular en la periferia urbana de Tuxtla Gutiérrez, Chiapas, México. Quehacer Científico en Chiapas (4), ISSN 1405-6542, pp 4-13.

Arias Guevara, Al. U., Fuentes Aguilera, J. I., Granados Mendoza, V. J. (2013). Control de calidad de las propiedades de resistencia a la compresión, absorción y peso volumétrico para las unidades de carga de mampostería, fabricados mediante procesos manuales y semi-industriales utilizando agregados de las canteras de Aramuaca y Ereguayquín de la Zona Oriental de El Salvador (Tesis de licenciatura). Facultad Multidisciplinaria Oriental, Universidad de El Salvador, San Miguel, El Salvador, Centroamérica.

ASTM C 1314, 2003. Standard Test Method for Compressive Strength of Masonry Prisms. United States of America.

ASTM C90-99a, 1999. Standard Specification for Loadbearing Concrete Masonry Units. United States of America.

Castro Hernández, P. A., Gaspar Vázquez, J. L., Palma Ordaz, N. (2009). Manual de fabricación de tabicón sólido de concreto (tesis de licenciatura). Unidad Profesional Interdisciplinaria de Ingeniería y Ciencias Sociales y Administrativas, Instituto Politécnico Nacional, México, Distrito Federal.

DoF (Diario Oficial de la Federación) (2014). Programa Nacional de Vivienda 2014-2018. Recuperado de http://dof.gob.mx/nota_detalle.php?codigo= $5342865 \&$ fecha $=30 / 04 / 2014$.

Fernández Baqueiro, L.; Marín Gómez, F.; Varela Rivera, J.; Vargas Marín, G. (2009). Determinación de la resistencia a compresión diagonal y el módulo de cortante de la mampostería de bloques huecos de concreto. Ingeniería, Revista Académica de la FI-UADY, 13-2, pp. 41-50.

INDECOPI, 2003. NTP 399.605. Método de ensayo para la determinación de la resistencia a compresión de prismas de albañilería. Perú.

Meli R. (1975). Comportamiento sísmico de muros de mampostería. Instituto de Ingeniería, unam, Serie Azul No. 352. México, DF, abril, 114 pp.

Morales Padilla, M. A. (2008). Evaluación de la resistencia a la compresión de blocks fabricados en la región de Perote Ver., de acuerdo a la norma NMXC-ONNCCE-2004 (Tesis de licenciatura). Facultad de Ingeniería Civil, Región Xalapa, Universidad Veracruzana, Xalapa de Enríquez, Veracruz.

Navas Carro, A.; Fonseca Mojica, C. (2016). Densidad de la mampostería de concreto en Costa Rica. Ingeniería 26 (2): 93-116, ISSN: 1409-2441; 2016. San José, Costa Rica. 
Normas Técnicas Complementarias para Diseño y Construcción de Estructuras de Mampostería. (2017). Gaceta Oficial del Distrito Federal. México.

ONNCCE. (2015). NMX-C-021-ONNCCE-2015. Organismo Nacional para la Normalización y Certificación para la Construcción y Edificación. México.

ONNCCE. (2013). NMX-C-036-ONNCCE-2013. Organismo Nacional para la Normalización y Certificación para la Construcción y Edificación. México. ONNCCE. (2013). NMX-C-038-ONNCCE-2013. Organismo Nacional para la Normalización y Certificación para la Construcción y Edificación. México.

ONNCCE. (2015). NMX-C-061-ONNCCE-2015. Organismo Nacional para la Normalización y Certificación para la Construcción y Edificación. México.

ONNCCE. (1997). NMX-C-077- ONNCCE-1997. Organismo Nacional para la Normalización y Certificación para la Construcción y Edificación. México.

ONNCCE. (2012). NMX-C-404-ONNCCE-2012. Organismo Nacional para la Normalización y Certificación para la Construcción y Edificación. México.

ONNCCE. (2017). NMX-C-414-ONNCCE-2017. Organismo Nacional para la Normalización y Certificación para la Construcción y Edificación. México.

ONNCCE. (2013). NMX-C-441-ONNCCE-2013. Organismo Nacional para la Normalización y Certificación para la Construcción y Edificación. México.

ONNCCE. (2010). NMX-C-464-ONNCCE-2010. Organismo Nacional para la Normalización y Certificación para la Construcción y Edificación. México.

Quiun Wong, D., San Bartolomé, A.; Mendoza, G. (2007). Corrección por esbeltez en pilas de albañilería ensayadas a compresión axial. Proyecto SEnCICO-PUCP. Pontificia Universidad Católica del Perú. Recuperado de http://blog.pucp.edu.pe/blog/wp-content/uploads/sites/82/2007/o4/ Esbeltez-en-Pilas.pdf

San Bartolomé, A.; Romero C.; Torres, J. C. (2007). Mejora de la adherencia bloque mortero. Pontificia Universidad Católica del Perú. Recuperado de http://blog.pucp.edu.pe/blog/wp-content/uploads/sites/82/2007/o4/ Adherencia-bloque-mortero.pdf

SENCICO, 2004. Proyecto de Norma Técnica de Edificación E.o7o "Albañilería". Comité Técnico de la Norma E.070. Perú.

Tena Colunga, A.; Artemio Juárez, A.; Salinas Vallejo, V. H. (2007). Resistencia y deformación de muros de mampostería combinada y confinada sujetos a cargas laterales. Revista de Ingeniería Sísmica No. 76, pp 29-60

Varela Rivera, J. L.; González Torres, V.; Fernández Baqueiro, L. E.; Vargas Marín, G. (2008). Determinación de la resistencia a compresión axial y el módulo de elasticidad de la mampostería de bloques huecos de concreto. XVI Congreso Nacional de Ingeniería Estructural. Sociedad Mexicana de Ingeniería Estructural, Veracruz, Veracruz (México). 